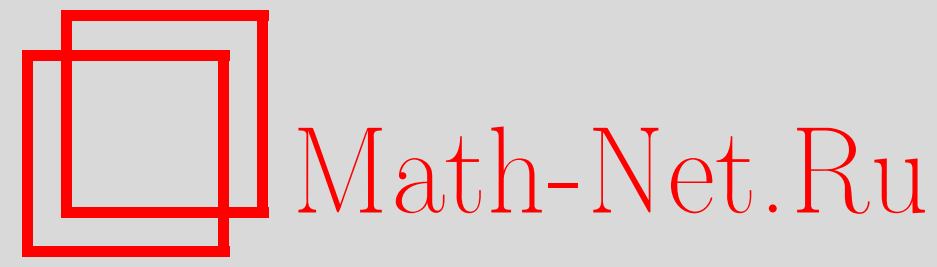

Н. В. Смородина, Асимптотическое разложение распределения однородного функционала от строго устойчивого случайного вектора. II, Теория вероятн. и ее примен., 1999, том 44, выпуск 2, 458-465

DOI: https://doi.org/10.4213/tvp783

Использование Общероссийского математического портала Math-Net.Ru подразумевает, что вы прочитали и согласны с пользовательским соглашением http://www . mathnet.ru/rus/agreement

Параметры загрузки:

IP : 18.234 .156 .22

26 апреля 2023 г., 15:59:45

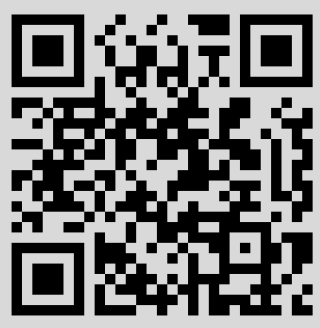


11. Embrechts P., Goldie C.M., Veraverbeke $N$. Subexponentiality and infinite divisibility. - Z. Wahrscheinlichkeitstheor. verw. Geb., 1979, B. 49, S. 335-347.

12. Embrechts P., Goldie C.M. On convolution tails. - Stochastic Process. Appl., 1982, v. 13, p. 263-278.

Поступила в редакцию 22.IX.1998

(C) $1999 \mathrm{r}$.

СМОРОДИНА Н. В.*

\title{
АСИМПТОТИЧЕСКОЕ РАЗЛОЖЕНИЕ \\ РАСПРЕДЕЛЕНИЯ ОДНОРОДНОГО ФУНКЦИОНАЛА ОТ СТРОГО УСТОЙЧИВОГО СЛУЧАЙНОГО ВЕКТОРА. ІІ ${ }^{1)}$
}

\begin{abstract}
Пусть $u$ - строго устойчивый негауссовский вектор с показателем устойчивости $\alpha \geqslant 1$, принимающий значения в сепарабельном банаховом пространстве $B$. Пусть $h: B \rightarrow \mathbf{R}-$ гладкий однородный функционал, и пусть $F-$ функция распределения случайной величины $h(u)$. Для функпии $1-F(x)$ получено асимптотическое разложение вида $\sum_{k=1}^{n} c_{k} x^{-k \alpha}+O\left(x^{-(n+1) \alpha}\right), x \rightarrow \infty,(n$ определяется гладкостью $h$ ). Для доказательства этого разложения использован новый подход, основанный на разложении распределения в сумму линейных функционалов.
\end{abstract}

Ключевые слова и фразы: строго устойчивое распределение, спектральная мера, пространство конфитурапий, пуассоновская случайная мера, линейный функционал на банаховом пространстве, стохастический интеграл.

Пусть $(B,\|\cdot\|)$ - сепарабельное банахово пространство, $u$ - строго устойчивый случайный вектор со значениями в $B, h: B \rightarrow[0, \infty)$ - гладкий однородный функционал. В работе автора [1] в случае, когда показатель устойчивости $\alpha$ вектора $u$ меньше единицы, для функции распределения (и ее производных) случайной величины $h(u)$ было получено асимптотическое разложение вида $(l \geqslant 0, x \rightarrow \infty)$

$$
\frac{d}{d x^{l}} \mathbf{P}(h(u)>x)=\sum_{k=1}^{n} \frac{c_{k, l}}{k !} x^{-k \alpha-l}+O\left(x^{-(n+1) \alpha-l}\right)
$$

(число $\boldsymbol{n}$ определяется степенью гладкости функционала $h$ ).

Для получения этого разложения в [1] был использован подход, связанный, с одной стороны, с известным представлением устойчивого случайного вектора. в виде стохастического интеграла по пуассоновской случайной мере, а с другой стороны, с разложением распределения (в пространстве реализаций) этой случайной меры в сумму линейных функционалов, каждый из которых хорошо приспособлен для выделения отдельных слагаемых асимптотики. Данное разложение распределения случайной меры является «пуассоновским» аналогом разложения винеровских функционалов в сумму кратных стохастических интегралов. Предположения о характере поведения интенсивности пуассоновской случайной меры, которые были использованы в [1] при получении этого разложения, оказались довольно ограничительными, в частности, позволили рассмотреть только устойчивые векторы с показателем устойчивости $\alpha<1$.

*C.-Петербургский государственный университет; e-mail: smorodin@ns2691.spb.edu

1) Работа выполнена при финансовой поддержке Российского фонда фундаментальных исследований, грант 96-15-96870. 
Настоящая работа является продолжением [1]. В ней получено аналогичное представление для распределения пуассоновской случайной меры при существенно иных предположениях об ее интенсивности. Это дало возможность получить асимптотическое разложение (1) для случая, когда показатель устойчивости $\alpha \in[1,2)$, а спектральная мера $\pi$ удовлетворяет условию $\int_{S} \theta \pi(d \theta)=0$, где $S=\{b \in B:\|b\|=1\}-$ единичная сфера в $B$. Используемые в настоящей работе обозначения и терминология соответствуют [1].

1. Необходимые определения. Пусть на пространстве $G=S \times R_{0}$, где $S$ полное сепарабельное метрическое пространство, а $R_{0}-$ полупрямая $(0, \infty)$ (далее символом $R_{e}$ будем обозначать полупрямую $(\varepsilon, \infty)$, а символом $G_{e}-$ множество $S \times$ $R_{e}$ ), задана пуассоновская случайная мера $\nu(d \theta, d x), \theta \in S, x \in R_{0}$, с интенсивностью $\Pi(d \theta, d x)=\pi(d \theta) \times \xi(d x)$, где $\pi(d \theta)-$ некоторая конечная мера на $S$. Предположим тахже, что мера $\Pi(d \theta, d x)$ удовлетворяет условию

$$
\int_{G}\left(x^{2} \wedge 1\right) \Pi(d \theta, d x)<\infty
$$

В точности так же, как в [1], мы будем всегда считать, что случайная мера $\nu(d \theta, d x)$ определена на вероятностном пространстве $(\mathfrak{X}, \Sigma, P)$, где $\mathfrak{X}=\mathfrak{X}(G)$ - пространство конфигураций на $G ; \Sigma-\sigma$-алгебра на $\mathfrak{X}(G)$ (см. [3]), $\mathbf{P}-$ соответствующщая $\nu$ вероятностная мера (распределение) в $\mathfrak{X}$. На вероятностном пространстве $(\mathfrak{X}, \Sigma, \mathbf{P})$ случайная мера $\nu$ задается естественной формулой $\nu(V, X)=\operatorname{card}(X \cap V)$, $V \subset G, X \in \mathfrak{X}(G)$.

Важное значение в наших рассуждениях будут иметь конечные конфигурация. Мы будем использовать обозначение $c=c_{k}$ для отображения $G^{k} \rightarrow \mathfrak{X}$ $c_{k}:\left(g_{1}, \ldots, g_{k}\right) \longmapsto\left\{g_{1}, \ldots, g_{k}\right\}$.

Сомножители $S$ и $R_{0}$ будут играть в дальнейшем различную роль. Пространству $S$ отводится роль вспомогательного параметра. Операции дифференцирования будут производиться только «вдоль $R_{0} 》 ;$ все встречающиеся в тексте значки производной по умолчанию соответствуют дифференцированию по $x \in R_{0}$, координата $\theta \in S$ при этом остается постоянной.

2. Представление меры $P$. В предположении, что интенсивность П удовлетворяет условню

$$
\int_{G}(x \wedge 1) \Pi(d \theta, d x)<\infty
$$

в [1] было получено представление для меры $P$ вида

$$
P=\sum_{k=0}^{\infty} \frac{1}{k !} \mu^{k} .
$$

В этом представлении $\mu^{0}=\delta_{\{\varnothing\}}-\delta$-мера с нагрузкой на пустой конфигурации,

$$
\mu^{1}(f)=\int_{G} \Delta f d \Pi, \quad \ldots, \quad \mu^{k}(f)=\int_{G^{k}} \Delta^{k} f d \Pi^{k}, \ldots,
$$

где $\Delta f(\theta, x)=\Delta^{1} f(\theta, x)=f(\theta, x)-f(\theta, 0)$ - приращение $f$ на $[0, x] ; \Delta^{k} f$ - аналогичное приращение на параллелепипеде $[0, x]$ размерности $k$. Область определения функционала $\mu^{k}$ включает $C^{k}$-гладкие по $x$ функции, ограниченные на $\bar{G}^{k}$ вместе с производными (по переменной $x$ ) до порядка $k$ включительно. Функционалы $\mu^{k}$ мы рассматриваем также и на множестве функций вида $f: \mathfrak{X} \rightarrow \mathbf{R}$, полагая по определению $\mu^{k}(f) \stackrel{\text { def }}{=} \mu^{k}\left(f_{k}\right)$, где $f_{k}=f \circ c_{k}$ - ограничение функции $f$ на $k$-точечные конфитурации.

Нетрудно видеть, что условие (1) не обеспечивает для рассматриваемого класса функций сходимости интегралов (3) и, соответственно, не может быть справедливо представление (2). Тем не менее, оказалось возможным, введя несколько другие функционалы $\widetilde{\mu}_{k}$, получить в данном случае аналог разложения (2)

$$
P=\sum_{k=0}^{\infty} \frac{1}{k !} \tilde{\mu}^{k} .
$$


Следует отметить, что хотя формально выражения (4) и (2) вполне аналогичны, область определения правой части (4) несколько уже, чем правой части (2).

Перейдем к непосредственному определению функционалов $\widetilde{\mu}^{k}$, входящих в разложение (4). Кақ и ранее, $\widetilde{\mu}^{0}=\mu^{0}=\delta_{\{\varnothing\}}$. Далее, для $f: G \rightarrow \mathbf{R}$ положим

$$
\tilde{\boldsymbol{\mu}}^{1}(f)=\int_{G} \tilde{\Delta} f d \Pi=\int_{S \times(0,1]} \tilde{\Delta} f d \Pi+\int_{S \times(1, \infty)} \tilde{\Delta} f d \Pi,
$$

где $\widetilde{\Delta} f(\theta, x)=f(\theta, x)-f(\theta, 0)-x f^{\prime}(\theta, 0) \chi_{[0,1]}(x)$. В последнем выражении $f^{\prime}-$ производная по $x$, а $\chi_{[0,1]}$ - индикаторная функция $[0,1]$. Для $k>1$ и $f: \bar{G}^{k} \rightarrow$ $\mathbf{R}$ положим $\widetilde{\mu}^{k}(f)=\int_{G^{k}} \widetilde{\Delta}^{k} f d \Pi^{k}$, где $\widetilde{\Delta}^{k}=\widetilde{\Delta}_{1} \ldots \widetilde{\Delta}_{k}$ (в последнем выражении $\Delta_{i}$ означает применение оператора $\Delta$ по переменной $\left.x_{i}\right)$. Очевидно, что $\widetilde{\mu}^{k}=\widetilde{\mu}_{1}^{1} \cdots \widetilde{\mu}_{k}^{1}$.

Как и выше, для функций $f: \mathfrak{X}(G) \rightarrow \mathbf{R}$ положим $\widetilde{\boldsymbol{\mu}}^{0}(f)=f(\varnothing)$ и $\widetilde{\boldsymbol{\mu}}^{k}(f)=\widetilde{\boldsymbol{\mu}}^{k}\left(f_{k}\right)$, где $f_{k}=f \circ c_{k}-$ сужение $f$ на $k$-точечные конфигурации.

Пусть $f(\bar{\theta}, \bar{x})$ - функция на $\bar{G}^{k}, I=\left\{i_{1}, \ldots, i_{j}\right\}$ - произвольное подмножество (возможно пустое) множества $1, \ldots, k,|I|=j$ - число элемен тов множества $I$. Будем обозначать символом $\partial_{I} f$ частную производную $\partial^{j} f /\left(\partial x_{i_{1}} \cdots \partial x_{i_{j}}\right)$, а символом $\partial_{I}^{2} f$ частную производную $\partial^{2 j} f /\left(\partial^{2} x_{i_{1}} \cdots \partial^{2} x_{i_{j}}\right)$.

Пусть далее $\tilde{\Delta}_{i}$ и $\int \cdot d \Pi_{i}$ обозначают соответственно операцию $\widetilde{\Delta}$ по переменной $x_{i}$ и интегрирование по $\left(\theta_{i}, x_{i}\right)$. Положим еще

$$
\widetilde{\Xi}=\int_{G}\left(\frac{x^{2}}{2} \wedge 2\right) \Pi(d \theta, d x)=\frac{1}{2} \int_{S \times(0,2]} x^{2} \Pi(d \theta, d x)+2 \int_{G_{2}} \Pi(d \theta, d x)=c_{1}+c_{0} .
$$

Будем говорить, что симметричная функция $f: \bar{G}^{k} \rightarrow \mathbf{R}$ принадлежит пространству $\widetilde{\Phi}_{b}^{k}$, если она $2 k$ раз дифференцируема по $\bar{x} \in(0, \infty)^{k}$, имеет конечную норму

$$
\|f\|_{k}=\max _{0 \leqslant i \leqslant k G^{k}} \sup _{\{1, \ldots, i\}} f \mid<\infty
$$

(в определении нормы $\|\cdot\|_{k}$ уже учтена симметричность) и

$$
\int_{S} \partial_{1} f\left(\theta_{1}, \ldots, \theta_{k} ; 0, x_{2}, \ldots, x_{k}\right) \pi\left(d \theta_{1}\right)=0 .
$$

Нам потребуется оценка нормы функционала $\widetilde{\mu}_{k}$ на введенном пространстве $\widetilde{\Phi}_{b}^{k}$.

Лемма 1. Пусть $f \in \widetilde{\Phi}_{b}^{k}$. Тогдa $\left|\widetilde{\mu}^{k}(f)\right| \leqslant \widetilde{\Xi}^{k}\|f\|_{k}$.

Д ок а з а те ль с т в о. Воспользуемся здесь тем, что в силу (6)

При $k=1$ имеем

$$
\int_{S \times(1,2]} x f^{\prime}(\theta, 0) d \pi=0
$$

$$
\begin{aligned}
\left|\tilde{\mu}^{1}(f)\right| \leqslant & \left|\int_{S \times(0,2]}\left(f(\theta, x)-f(\theta, 0)-x f^{\prime}(\theta, 0)\right) d \Pi\right| \\
& +\int_{G_{2}}|f(\theta, x)-f(\theta, 0)| d \Pi \leqslant\|f\|_{1} c_{1}+\|f\|_{1} c_{0}=\|f\|_{1} \widetilde{\Xi} .
\end{aligned}
$$

При $k=2$

$$
\begin{aligned}
\left|\tilde{\mu}^{2}(f)\right| \leqslant & \left|\int_{G_{2}} \tilde{\Delta}_{1} \tilde{\Delta}_{2} f d \Pi_{2} d \Pi_{1}\right| \leqslant \int_{S \times(0,2)}\left|\tilde{\Delta}_{1} \int_{G} \widetilde{\Delta}_{2} f d \Pi_{2}\right| d \Pi_{1} \mid \\
& +\int_{G_{2}}\left|\Delta_{1} \int_{G} \tilde{\Delta}_{2} f d \Pi_{2}\right| d \Pi_{1} \leqslant c_{1}\left\|\partial_{\{1\}}^{2} \int_{G} \tilde{\Delta}_{2} f d \Pi_{2}\right\|_{\infty}+c_{0}\left\|\int_{G} \widetilde{\Delta}_{2} f d \Pi_{2}\right\|_{\infty} \\
\leqslant & c_{1}\left\|\int_{S \times(0,2]} \widetilde{\Delta}_{2} \partial_{\{1\}}^{2} f d \Pi_{2}+\int_{S \times R_{2}} \Delta_{2} \partial_{\{1\}}^{2} f d \Pi_{2}\right\|_{\infty}+c_{0} \widetilde{\Xi}_{\|}\|f\|_{2} \\
\leqslant & \left(c_{1}+c_{0}\right) \widetilde{\Xi}\|f\|_{2}=\widetilde{\Xi}^{2}\|f\|_{2} .
\end{aligned}
$$


Далее следует элементарная индукция по $k$ :

$$
\begin{aligned}
\left|\tilde{\mu}^{k}(f)\right|= & \left|\int_{G^{k}} \tilde{\Delta}_{1} \cdots \tilde{\Delta}_{k} f d \Pi_{k} \cdots d \Pi_{1}\right| \leqslant c_{1}\left\|\partial_{\{1\}}^{2} \int_{G^{k-1}} \tilde{\Delta}_{2} \cdots \tilde{\Delta}_{k} f d \Pi_{k} \cdots d \Pi_{2}\right\|_{\infty} \\
& +c_{0}\left\|\int_{G^{k-1}} \tilde{\Delta}_{2} \cdots \widetilde{\Delta}_{k} f d \Pi_{k} \cdots d \Pi_{2}\right\|_{\infty} \\
\leqslant & \cdots \leqslant \sum_{I \subset\{1, \ldots, k\}} c_{1}^{|I|} c_{0}^{k-|I|}\left\|\partial_{I}^{2} f\right\|_{\infty} \leqslant\left(c_{1}+c_{0}\right)^{k}\|f\|_{k}=\widetilde{\Xi}^{k}\|f\|_{k} .
\end{aligned}
$$

Перейдем теперь непосредственно к выводу разложения (4). Пусть функция $f: \mathfrak{X} \rightarrow \mathbf{R}$ такова, что для любого $k$ функиия $f_{k} \in \widetilde{\Phi}_{b}^{k}$ (в частности, продолжается надлежащим образом на $\bar{G}^{k}$ и удовлетворяет (6)). Положим $\|f\| \stackrel{\text { def }}{=} \sum_{k=0}^{\infty} \widetilde{\Xi}^{k}\left\|f_{k}\right\|_{k} / k$ !.

Введем пространство $F A_{s}$ функций $f: \mathfrak{X} \rightarrow \mathbf{R}$, обладающих свойствами:

1) $\|f\|<\infty$;

2) каждая из фунхций $f_{k}(k \geqslant 0)$ является граничным значением «следующей» функции $f_{k+1}$, в том смысле, что $f_{k+1}\left(\theta_{1}, \ldots, \theta_{k}, \theta_{k+1}, x_{1}, \ldots, x_{k}, 0\right)=$ $f_{k}\left(\theta_{1}, \ldots, \theta_{k}, x_{1}, \ldots, x_{k}\right)$;

3) последовательность функций $f_{\varepsilon}(X)=f\left(X \cap G_{\varepsilon}\right)$, где $G_{\varepsilon}=S \times(\varepsilon, \infty)$, сходится по мере $P$ к функции $f(X)$.

Теперь сформулируем утверждение, даютее нам разложение (4).

Теорема 1. Пусть $f \in F A_{s}$. Тогда

$$
\int f d P=\sum_{k=0}^{\infty} \frac{1}{k !} \tilde{\mu}^{k}(f) \text {. }
$$

Д о к аз за те ль с т в о. Положим $f_{e}(X)=f\left(X \cap G_{\varepsilon}\right)$. Очевидно, что, доказав (7) для функций $f_{\varepsilon}$ и перейдя к пределу при $\varepsilon \rightarrow 0$, мы получим (7) в полном объеме. В силу (2) (теорема 2 из [1]) имеем

$$
\int f_{e} d P=\sum_{k=0}^{\infty} \frac{1}{k !} \int_{G_{\varepsilon}^{k}} \Delta^{k} f_{k} d \Pi^{k} .
$$

Теперь остается только заметить, что в силу (6) для любого $k \geqslant 0$

$$
\int_{G_{c}^{k}} \Delta^{k} f_{k} d \Pi^{k}=\int_{G_{\varepsilon}^{k}} \widetilde{\Delta}^{k} f_{k} d \Pi^{k}
$$

Теорема 1 полностью доказана.

Заметим, что, по сравнению с условием аналогичной теоремы из [1], новым в данном случае является условие (6), которое в некотором смысле является условием симметрии. Например, при изучении функционалов от устойчивых вехторов, когда в качестве $S$ выбирается единичная сфера в банаховом пространстве, $\Pi(d \theta, d x)=$ $\pi(d \theta) d x / x^{1+\alpha}(\pi$ - спектральная мера устойчивого вектора), а класс необходимых функций $f$ есть гладкие (по Фреше) функции от стохастического интеграла вида

$$
f(X)=\phi\left(\lim _{\varepsilon \rightarrow 0}\left(\sum_{(\theta, x) \in X \cap G_{\varepsilon}} \theta x-\int_{G_{\varepsilon}} \theta x d \Pi\right)\right),
$$

для справедливости (6) достаточно выполнения условия $\int_{S} \theta \pi(d \theta)=0$.

3. Представление остаточного члена. Кроме разложения (2), требующего неограниченной гладкости функции, к которой применяется функционал $P$, в [1] получено также представление $P=\sum_{k=0}^{n} \frac{1}{k !} \mu^{k}+r_{n}$, для остаточного члена $r_{n}$ которого получено выражение, не требующее неограниченной гладкости. Отметим, что именно это представление было использовано в [1] для получения асимптотических разложений.

В настоящем пункте будет получено разложение «с остаточным членом» для представления (4). 
Рассмотрим величину $r_{n}(f, \varepsilon)=\sum_{k=n+1}^{\infty} \int_{G_{\varepsilon}^{k}} \widetilde{\Delta}^{k} f_{k} d \Pi^{k}$ и получим для нее выражение, включающее лишь разности не выше $(n+1)$-го порядха. В силу (6) имеем $r_{n}(f, \varepsilon)=\sum_{k=n+1}^{\infty} \int_{G_{\varepsilon}^{k}} \Delta^{k} f_{k} d \Pi^{k}$. Пользуясь представлением (15) из [1], а затем снова (6), получаем

$$
r_{n}(f, \varepsilon)=e^{-\Pi\left(G_{\varepsilon}\right)} \sum_{t=1}^{\infty} A_{t}\left(G_{\varepsilon}\right) \int_{G_{\varepsilon}^{t+n}} \widetilde{\Delta}_{\{1, \ldots, n+1\}} f_{t+n} d \Pi^{t+n}
$$

где

$$
A_{t}\left(G_{\varepsilon}\right)=\frac{1}{n !} \sum_{k=0}^{\infty} \frac{(k+n) !}{(k+n+t) !} \frac{\Pi\left(G_{\varepsilon}\right)^{k}}{k !} .
$$

Для перехода к пределу при $\varepsilon \rightarrow 0$ в выражении (8) необходимы определенные предположения о функции $f$. Пусть сначала $g-$ функция на $\bar{G}^{k}$, симметричная относительно перестановок сомножителей $G$.

Зафиксируем $n \leqslant k$ и определим норму $\|g\|_{k, n}$ формулой

$$
\|g\|_{k, n}=\max _{0 \leqslant i \leqslant n} \sup _{G^{k}}\left|\partial_{\{1, \ldots, i\}}^{2} g\right| .
$$

Пусть теперь $f: \mathfrak{X} \rightarrow \mathbf{R}-$ функция на пространстве конфигураций. Будем говорить, что $f$ принадлежит пространству $F A_{s}(n)\left(f \in F A_{s}(n)\right)$, если

$$
\|f\|_{F A_{,}(n)} \stackrel{\text { def }}{=} \sup _{k \geqslant n}\left\|f_{k}\right\|_{k, n}<\infty,
$$

выполнены требования 2) и 3) из определения пространства $F A_{s}$, а также условие (6).

Из леммы 1 следует, что для $f \in F A_{s}(n+1)$

$$
\left|\int_{G_{c}^{t+n}} \widetilde{\Delta}_{\{1, \ldots, n+1\}} f_{t+n} d \Pi^{t+n}\right| \leqslant \widetilde{\Xi}^{n+1}\left(\Pi\left(G_{e}\right)\right)^{t-1}\|f\|_{t+n, n},
$$

так что $\left|r_{n}(f, \varepsilon)\right| \leqslant \widetilde{\Xi}^{n+1} e^{-\Pi\left(G_{\varepsilon}\right)} \sum_{t=1}^{\infty} A_{t}\left(G_{e}\right)\left(\Pi\left(G_{e}\right)\right)^{t-1}\|f\|_{F A_{s}(n+1)}$, при этом

$$
\begin{aligned}
\sum_{t=1}^{\infty} A_{t}\left(G_{e}\right) \Pi\left(G_{e}\right)^{t-1} & =\frac{1}{n !} \sum_{t=1}^{\infty} \sum_{k=1}^{\infty} \frac{(k+n) !}{(k+n+t) ! k !} \Pi\left(G_{e}\right)^{k+t-1}=(k+t-1=s) \\
& =\sum_{s=0}^{\infty} \frac{\Pi\left(G_{e}\right)^{s}}{(s+n+1) !} \cdot \sum_{k=0}^{s} \frac{(k+n) !}{n ! k !}=\frac{1}{(n+1) !} e^{\Pi\left(G_{s}\right)} .
\end{aligned}
$$

На последнем шаге мы воспользовались комбинаторным тождеством $\sum_{k=0}^{s}(k+$ $n) ! /(n ! k !)=(s+n+1) ! /((n+1) ! s !)$. Окончательно получаем

$$
\left|r_{n}(f, \varepsilon)\right| \leqslant \frac{\widetilde{\Xi}^{n+1}}{(n+1) !}\|f\|_{F A_{s}(n+1)} .
$$

Из этой оценки вытекает существование предела $r_{n}(f)=\lim _{\varepsilon \rightarrow 0} r_{n}(f, \varepsilon)$, также удовлетворяющего (10).

Представление (8) и вытекающее из него представление для $r_{n}(f)$ допускают удобную модификацию. Именно, по тем переменным, по которым берется оператор $\widetilde{\Delta}$, область интегрирования может быть заменена на махсимальную, т.е. на $G^{n+1}$.

Теорема 2. Пусть $f \in F A_{s}(n+1)$. Тогда

$$
r_{n}(f)=\lim _{\varepsilon \rightarrow 0} e^{-\Pi\left(G_{c}\right)} \sum_{t=1}^{\infty} A_{t}\left(G_{e}\right) \int_{G_{c}^{t-1}} \widetilde{\mu}_{\{1, \ldots, n+1\}}\left(f_{t+n}\right) d \Pi^{\{n+2, \ldots, n+t\}} .
$$

Для доказательства нам необходимо оденить интеграл вида $\left|\int_{G^{n}-G_{\varepsilon}^{n}} \widetilde{\Delta}^{n} g d \Pi^{n}\right|$. Для $I \subset\{1, \ldots, n\}$ положим $G_{i}=S \times(0, \varepsilon)$ при $i \in I, G_{i}=S \times R_{\text {в }}$ при $і \in C I$ и определим $G_{I}=\prod_{i=1}^{n} G_{i}$. 
Очевидно, что $G^{n}-G_{\varepsilon}^{n}=\cup_{I \neq \varnothing} G_{I}$. Далее, рассуждения, аналогичные используемым при доказательстве леммы 1 , приводят нас к оценке

$$
\left|\int_{G_{I}} \widetilde{\Delta}^{n} g d \Pi^{n}\right|=\left|\int_{G_{I}} \widetilde{\Delta}^{I} \widetilde{\Delta}_{C I} g d \Pi^{n}\right| \leqslant(\Xi(S \times(0, \varepsilon]))^{|I|} \widetilde{\Xi}\left(G_{\varepsilon}\right)^{n-|I|}\|g\|_{n, n},
$$

где $\widetilde{\Xi}(B)=\int_{B}\left(x^{2} / 2 \wedge 2\right) \Pi(d \theta, d x)$.

Суммируя по $I$, получаем

$$
\left|\int_{G^{n}-G_{\varepsilon}^{n}} \widetilde{\Delta}^{n} g d \Pi^{n}\right| \leqslant\left(\widetilde{\Xi}^{n}-\left(\widetilde{\Xi}\left(G_{e}\right)\right)^{n}\right)\|g\|_{n, n} \leqslant n \tilde{\Xi}(S \times(0, \varepsilon]) \widetilde{\Xi}^{n-1}\|g\|_{n, n} .
$$

Из сравнения (8) и (11) видно, что достаточно доказать сходимость к нулю при $\varepsilon \rightarrow 0$ выражения вида

$$
\begin{aligned}
& e^{-\Pi\left(G_{\varepsilon}\right)} \sum_{t=1}^{\infty} A_{t}\left(G_{\varepsilon}\right) \int_{G_{\varepsilon}^{t-1}} \mid \int_{G_{\varepsilon}^{n+1}} \widetilde{\Delta}_{\{1, \ldots, n\}} f_{t+n} d \Pi^{\{1, \ldots, n+1\}} \\
& \qquad-\widetilde{\mu}_{\{1, \ldots, n+1\}}\left(f_{t+n}\right) \mid d \Pi^{\{n+2, \ldots, n+t\}} \\
& \leqslant e^{-\Pi\left(G_{*}\right)} \sum_{t=1}^{\infty} A_{t}\left(G_{\varepsilon}\right) \Pi\left(G_{\varepsilon}\right)^{t-1} \widetilde{\Xi}^{n}(n+1) \Xi(S \times(0, \varepsilon])\left\|f_{n+t}\right\|_{n+t, n+1} \\
& \leqslant\|f\|_{F A_{-}(n+1)} \frac{\widetilde{\Xi}_{n !}^{n}}{n !}(S \times(0, \varepsilon]) \longrightarrow 0, \quad \varepsilon \rightarrow 0 .
\end{aligned}
$$

На последнем шаге преобразований мы воспользовались (9).

4. Асимптотическое разложение распределения однородного функционала от строго устойчивого случайного вектора. Пусть $u$ - строго устойчивый случайный вектор с показателем устойчивости $1 \leqslant \alpha<2$, принимающий значения в селарабельном банаховом пространстве $B$. В точности так же, как в [1], положим $G=S \times R_{0}$, где $S$ - единичная сфера банахового пространства, $\xi(d x)=d x / x^{1+\alpha}$, $\Pi(d \theta, d x)=\pi(d \theta) d x / x^{1+\alpha}$, где $\pi$ - спектральная мера вектора $u$. Мы предполагаем, что мера $\pi$ удовлетворяет условию

$$
\int_{S} \theta \pi(d \theta)=0
$$

а вектор $u$ на вероятностном пространстве $(\mathfrak{X}(S \times(0, \infty)), P)$ задается отображением $u(X)=\lim _{\varepsilon \rightarrow 0} \sum_{(\theta, x) \in X, x \geqslant \varepsilon} \theta x$. Последнее условне существенно только для $\alpha=1$, для $\alpha>1$ оно есть следствие строгой устойчивости (см. [4]).

Далее, пусть $h: B \rightarrow[0, \infty)$ - гладкий однородный функционал на $B$. Нас будет интересовать асимптотика функщии распределения и ее производных случайной величины $h(u)$. Сформулируем условия, которые мы будем накладывать на однородный функционал $h$. Именно, мы фиксируем $n \geqslant 2$ и требуем, чтобы функционал $h$ был $n$ раз дифференицируем по Фреше вне $h^{-1}(0)$, а его производные $\nabla^{(k)} h$, рассматриваемые как полилинейные функции, ограничены в следующем смысле:

$$
s(h) \stackrel{\text { def }}{=} \max _{k \leqslant n} \sup _{\theta_{1}, \ldots, \theta_{k} \in S} \sup _{b \in h^{-1}(0)}\left|\nabla^{(k)} h(b)\left[\theta_{1}, \ldots, \theta_{k}\right]\right|<\infty .
$$

Условие (13) мы называем условием $S U B(n)$.

Положим $H(X)=h(u(X))$. В силу (4) имеем

$$
P H^{-1}=\sum_{k=0}^{n} \frac{\tilde{\mu}^{k} H^{-1}}{k !}+r_{n} H^{-1},
$$

где функционалы $\widetilde{\mu}^{k} H^{-1}$ и $r_{n} H^{-1}$ определяются естественными соотношениями $\left(\tilde{\mu}^{k} H^{-1}\right)(f)=\tilde{\mu}^{k}(f \circ H), r_{n} H^{-1}(f)=r_{n}(f \circ H)$. Левая часть (14) есть распределение случайной величины $h(u)$, правая часть есть сумма линейных функционалов. 
Ниже будет показано, что при определенных предположениях функционалы $\widetilde{\mu}^{k} H^{-1}$ и $r_{n} H^{-1}$ являются мерами. Более того, однородность $h$ и степенной вид плотности меры $\xi$ приводят при $x>0$ к простой формуле

$$
\widetilde{\boldsymbol{\mu}}^{k} H^{-1}([x, \infty))=c_{k} x^{-k \alpha},
$$

откуда

$$
P(h(u)>x)=\sum_{k=1}^{n} \frac{c_{k}}{k !} x^{-k a}+\left(r_{n} H^{-1}\right)([x, \infty)) .
$$

Следуюцие две теоремы дают достаточные условия справедливости (15) и оценку остаточного члена в (16).

Теорема 3. Предположим, ито $h \in L^{\alpha}(S, d \pi)$ и удовлетворяет условию $S U B(2 n)$. Тогда функционалы $\widetilde{\mu}^{k} H^{-1}, k=1, \ldots, n$, ограниченкые ка $(0, \infty)$, явлнются мерами (положителькыми ияи отричательными) өида

$$
\left(\tilde{\mu}^{k} H^{-1}\right)([x, \infty))=c_{k} x^{-k \alpha}, \quad x>0 .
$$

Из теоремы 3 немедленно следует, что ограничение функционала $r_{n} H^{-1}$ является зарядом $\rho_{n}$, некоторые его свойства описывает теорема 4.

Теорема 4. Предположим, что $h \in L^{\alpha}(S, d \pi)$ и при мекотором $N \geqslant 0$ удовлетворяет условию $S U B(2 N+4 n+4)$. Тогда прочзводные $\rho_{n}^{(j)}$ заряда $\rho_{n}$, помимаеміые в смысле обобщенных фуякций, являются комечными зарядами, причем

$$
\left|\rho_{n}^{(j)}([x, \infty))\right| \leqslant \frac{\text { const }}{x^{(n+1) \alpha+j}}, \quad 0 \leqslant j \leqslant N .
$$

Следствие. Если $h \in L^{\alpha}(S, d \pi)$ и удовлетворяет условия $S U B(2 N+4 n+4)$ для мекоторого $N \geqslant 0$, mo $\partial_{\text {лg }}$ всех $0 \leqslant j \leqslant N$

$$
\frac{d^{j}}{d x^{j}} P(h(u) \geqslant x)=\sum_{k=1}^{n} \frac{c_{k, j}}{k !} x^{-k \alpha-j}+O\left(x^{-(n+1) \alpha-j}\right) .
$$

Доказательство теоремы 3 практически дословно повторяет доказательство теоремы 3 из [1], единственная разница - в количестве интегрирований по частям. В случае $\alpha \geqslant 1$ их требуется ровно вдвое больше, и связано это с тем, что в оценку для функционала $\widetilde{\mu}$ (лемма 1) входят не первые производные, а вторые. Соответственно, это приводит к вдвое большим требованиям гладкости для $h$.

Доказательство теоремы 4 также в большей своей части повторяет соответствуюшие рассуждения из [1, теорема 4], однако здесь имеется ряд различий. Мы опишем здесь только эти различия, опустив полностью совпадающую часть рассуждений.

При оценке остаточного члена в [1] использовалось его представление в виде суммы двух слагаемых $r_{n} H^{-1}(\phi)=r_{(1)}(\phi)+r_{(2)}(\phi)$. Для функщионала $r_{(2)}$ было доказано неравенство

$$
\left|r_{(2)}\left(\phi^{(N)}\right)\right| \leqslant \text { const }\|\phi\| E^{-N-(n+1) \alpha},
$$

$\phi-$ произвольная гладкая финитная функция с носителем в $(E, \infty)$, обеспечивающее степенную оценку хвоста $r_{(2)}$ (и его производных). Эта часть рассуждений в нашем случае может быть дословно повторена. Далее было показано, что заряд $r_{(1)}$ (и все его производные) имеет моменты любого порядка, что обеспечивает сверхстепенное убывание его хвоста. В этой части рассуждений имеются некоторые различия, далее мы их перечислим.

Различия появляются, начиная с формулы (35). В формуле перед ней не нужно оценивать $\left(1+\left\|u_{1}\right\|\right)^{L}$ через $\left(1+\sum_{i=n+2}^{n+1+l} x_{i}\right)^{L}\left(\operatorname{Tak}\right.$ как $\int_{0}^{2} x d x / x^{1+\alpha}=\infty$, это бесполезно). Соответственно, вместо выражения (35) нам придется доказать конечность выражения

$$
\lim _{\varepsilon \rightarrow 0} e^{-\Pi\left(G_{\varepsilon}\right)} \sum_{l=1}^{\infty} \sum_{m=0}^{\infty}\left(\begin{array}{c}
l+m \\
m
\end{array}\right) A_{l+m+1}\left(G_{\varepsilon}\right) \Pi\left(G_{2}\right)^{m} \int_{\left(G_{\varepsilon}-G_{2}\right)^{l}}\left\|\theta_{1} x_{1}+\cdots+\theta_{l} x_{l}\right\| d \Pi\left(G_{2}\right)^{l}
$$


Последующие выкладки вплоть до (37) никак не затрагивают самый внутренний интеграл. Соответственно, последнее выражение (вместо (37)) равно (множество $G_{e}-G_{2}$ мы обозначаем $\left.V_{e}\right)$

$$
\begin{aligned}
& \lim _{\varepsilon \rightarrow 0} e^{-\Pi\left(G_{e}-G_{2}\right)} \sum_{l=1}^{\infty} \sum_{i=0}^{\infty} \frac{\Pi\left(G_{e}-G_{2}\right)^{i}}{(i+l+1) !} \int_{\left(G_{e}-G_{2}\right)^{l}}\left\|\theta_{1} x_{1}+\cdots+\theta_{l} x_{l}\right\|^{L} d \Pi^{l} \\
& =\lim _{e \rightarrow 0} e^{-\Pi\left(V_{e}\right)} \sum_{l=1}^{\infty} \sum_{i=0}^{\infty} \frac{1}{(i+l+1) !} \int_{V_{c}^{l+i}}\left\|\theta_{1} x_{1}+\cdots+\theta_{l} x_{l}\right\|^{L} d \Pi^{l+i}=(i+l=k) \\
& =\lim _{e \rightarrow 0} e^{-\Pi\left(V_{e}\right)} \sum_{k=1}^{\infty} \sum_{i=0}^{k-1} \frac{1}{(k+1) !} \int_{V_{e}^{k}}\left\|\theta_{1} x_{1}+\cdots+\theta_{k-i} x_{k-i}\right\|^{L} d \Pi^{k}
\end{aligned}
$$

Воспользуемся теперь следуюшим хорошо известным неравенством [6].

Лемма 2. Пусть $X, Y$ - независимые $B$-зяачные случайные векторы, $X \in$ $L^{1}(B), \mathbf{E} X=0$. Пусть $f: \mathbf{R}_{+} \rightarrow \mathbf{R}$ - возрастаючая быпуклая фуякчия такая, umo E $f(\|X\|)<\infty$ u E $f(\|Y\|)<\infty$. Tогдa E $f(\|Y\|) \leqslant \mathbf{E} f(\|X+Y\|)$.

В силу этой леммы имеем

$$
\int_{V_{e}^{k}}\left\|\theta_{1} x_{1}+\cdots+\theta_{k-i} x_{k-i}\right\|^{L} d \Pi^{k} \leqslant \int_{V_{c}^{k}}\left\|\theta_{1} x_{1}+\cdots+\theta_{k} x_{k}\right\|^{L} d \Pi^{k}
$$

соответственно, правая часть (17) может быть оценена сверху через

$$
\begin{gathered}
\lim _{\varepsilon \rightarrow 0} e^{-\Pi\left(V_{\varepsilon}\right)} \sum_{k=1}^{\infty} \frac{1}{k !} \int_{V_{\varepsilon}^{k}}\left\|\theta_{1} x_{1}+\cdots+\theta_{k} x_{k}\right\|^{L} d \Pi^{k} \\
=\lim _{\varepsilon \rightarrow 0} \mathbf{E}\left\|\int_{V_{c}} \theta x d \nu\right\|^{L} \leqslant \mathbf{E}\left\|\int_{S \times(0,2]} \theta x d \nu\right\|^{L}
\end{gathered}
$$

где $\nu$ - пуассоновская случайная мера на $S \times(0,2]$ с интенсивностью П. Последняя величнна конечна, тақ как известно [6], что безгранично делимые распределения с конечным спектром имеют моменты любого порядка.

Этой оценкой и завершается доказательство теоремы 4.

\section{СПИСОК ЛИТЕРАТУРЫ}

1. Смородина H. B. Асимптотическое разложение однородного функционала от строго устойчивого случайного вектора. - Теория вероятн. и ее примен., 1996, т. 41, B. 1, c. 133-163.

2. Major $P$. Multiple Wiener-Itô Integral. Berlin: Springer-Verlag, 1981.

3. Керстан $\breve{~}$., Матmес К., Мекке Й. Безгранично делимые точечные процессы. M.: Наука, 1982. $392 \mathrm{c}$.

4. Linde $W$. Infinitely Divisible and Stable Measures on Banach Spaces. Leipzig: Teubner, 1983. $201 \mathrm{p}$.

5. Acosta A.de. Exponential moments of vector valued random series and triangular arrays. - Ann. Probab., 1980, v. 8, № 2, p. 381-389.

6. Юринский В. В. О безгранично делимых распределениях. - Теория вероятн. и ее примен., 1974, т. XIX, в. 2, с. 308-318. 